\title{
SISTEMAS Y MODELOS DE JUSTICIA CONSTITUCIONAL A LOS ALBORES DEL SIGLO XXI
}

\author{
Luca Mezzetti ${ }^{1}$ \\ Catedrático de Derecho Constitucional \\ Facultad de Derecho \\ Alma Mater Studiorum \\ Universidad de Bolonia (Italia)
}

La clasificación de los sistemas y modelos de justicia constitucional en el derecho comparado ha sido propuesta por acreditados estudiosos de culturas jurídicas diferentes: pueden acordarse, entre otras, las obras de Calamandrei, Cappelletti, Garcia Belaunde, Pegoraro, Ruggero y Spadaro, Weberio, Harutyunyan y Maucic, Vanossi, Sagüés, Brewer-Carias, Nogueira, Von Brünneck, Favoreu, Pasquino, a las cuales se hace ideal remisión por la originalidad que singularmente las caracteriza.

Una significativa tentativa de superación de la tipología tradicional de los modelos de control de constitucionalidad, de particular interés en cuanto llevada a cabo por un estudioso de cultura anglosajona, es atribuible a la teoría de E. Mcwhinney.

En su clásico estudio sobre el control de constitucionalidad en las experiencias jurídicas de matriz anglosajona [E. Mcwhinney, 1956] y en seno a estudio siguiente concerniente a la experiencia alemana de justicia constitucional [E. Mcwhinney, 1962], el autor utilizó la noción de judicial review de modo genérico y promiscuo para la individuación, en el primer caso, del papel desarrollado por los tribunales supremos en las materias de

1 El profesor Luca Mezzetti nos ha enviado el presente trabajo en septiembre de 2009, el cual por su interés académico, lo publicamos en nuestra sección documentos, con algunas adaptaciones formales a nuestra revista. 
relevancia constitucional frente a los otros poderes estatales $\mathrm{y}$, en el segundo, para describir la praxis del órgano de justicia constitucional aleman durante la aplicación de normas constitucionales y el escrutinio de los actos legislativos y ejecutivos, en este última hipotesis aplicando la noción misma a un contexto constitucional europeo-continental parte de la familia jurídica de civil law, si bien intensamente influenzado, en la fase posterior a la Segunda Guerra Mundial, por el derecho constitucional norteamericano. En el ámbito de obra más reciente [E. Mcwhinney, 1986], el constitucionalista canadiense subraya las limitaciones que manifiestan los dos idealtipos o modelos de clasificación representados por el judicial review de tipo americano y del constitutional review de tipo europeo-continental: eso en consideración de la mutua influencia que los modelos jurídicos si bien caracterizados por una diferente matriz histórica y cultural manifiestan de realizar en sus recíprocas interrelaciones y de la tendencia que el funcionalismo produce en seno al derecho constitucional de los diversos ordenamientos en la dirección de la asimilación de las respuestas institucionales y procedurales provistas por los sistemas constitucionales a problemáticas de idéntica u análoga naturaleza.

En tal sentido, evidente es según el autor la progresiva transformación de facto de los tribunales supremos operantes en los sistemas de common law en cortes o tribunales constitucionales especiales tales de denotar crecientes paralelismos con respecto de los modelos europeo-continentales de la familia romano-germánica. Los conflictos interorganicos e intesubjetivos forman parte actualmente de modo natural e indefectible en la normal actividad de jurisdicción constitucional desarrollada por los tribunales supremos, sean ellos de jure o de facto cortos constitucionales; todo los tribunales supremos, además, se pronuncian acerca de la interpretación y aplicación de los actos legislativos y administrativos en casos concretos, asimismo sobre los recursos de los individuos que lamentan la violación de sus propios derechos fundamentales. Aunque reconociendo la eventualidad que el control de constitucionalidad sea ejercido por órganos diferentes de una corte constitucional stricto sensu, como en el caso del Consejo constitucional francés, e incluso asumiendo las funciones de los órganos de justicia constitucional, en seno a los varios ordenamientos, dimensiones cuantitativas y cualitativas de variable entidad, Mcwhinney observa cómo el proceso de sustancial convergencia entre los dos modelos clásicos, en precedencia observada, sugiera una superación de la yuxtaposición judicial review-constitutional review para lograr un contexto analítico de referencia monístico y unitario, cuyas venas deben localizarse sobre la base de criterios estrechamente empíricos.

Tomando acto del aproche tradicional que, para el estudio de las diferentes tendencias seguidas por la justicia constitucional, acoge cual base de referencia los dos modelos del judicial review de matriz norteamericana y la Verfassungsgerichtsbarkeit de inspiración kelseniana, y constatado el proceso de progresiva convergencia de los modelos mismos, F. Rubio LLorente auspicia análogamente la superación de la mencionada yuxtaposición, fruto de un método de razonamiento o estudio preferiblemente de abandonar sea en razón de la recordada asimilación de los modelos que a causa de la diversidad de los sistemas europeos: resulta veleidoso y evanescente, segun el constitucionalista español, la referencia a un modelo europeo si se considera que las diferencias 
entre los sistemas europeos se revelan a veces de entidad mayor con respecto de aquellas entre el modelo europeo y el modelo americano [F. Rubio Llorente, 1996, 11 ss.].

Una razón de alcance todavía mayor se suma a tal orden de consideraciones: la categorización en dos grandes modelos contribuye a excluir del análisis las diferencias orgánicas y procedurales, incluso a omitir las realidades fundamentales, es decir las finalidades propias que atribuyen a la justicia constitucional su razón de ser, verdadero núcleo duro del instituto.

Es sobre la base de tales premisas que, en lugar de los usuales modelos de justicia constitucional, F. Rubio Llorente prefiere recurrir a la contraposición basada en los rasgos específicos de los mismos, sobre su centro de interés principal: el modelo que tiende a asegurar la constitucionalidad de la ley y el modelo que tiende a garantizar la efectividad de los derechos fundamentales.

El modelo basado sobre la ley se caracteriza principalmente por la presencia del recurso abstracto de constitucionalidad, o en vía principal. El modelo basado sobre la tutela de los derechos fundamentales demuestra de preferir el recurso concreto de constitucionalidad (o en vía incidental) y los instrumentos puestos a protección de las libertades (recurso de amparo, Verfassungsbeschwerde, etcétera).

Vienen en relieve peculiar, si bien no exclusivo, en seno al primero de los modelos propuestos, los recursos que el autor articuló anteriormente, en el ámbito de otra obra [F. RUBIO LLORENTE, 1992, 9 ss.], en objetivos, en cuanto no finalizados a la tutela de intereses propios de los recurrentes, y conflictivos, caracterizados en cambio por tal objetivo. Estos últimos pueden ser presentados, en línea de principio, por órganos u entes dotados de propias competencias, los unos y las otras contemplados directamente por la Constitución sin la necesaria intervención de la ley: con tal tipo de recursos la contestación de la ley se realiza en términos puramente abstractos, es decir sin tener en cuenta su concreta aplicación ni de la interpretación provista por el juez ordinario. Lo que se discute a tal respeto es la conformidad a la Constitución de dos concepciones políticas opuestas, la una -aquella al origen del recurso- que niega la legitimidad constitucional de aquella sustentada por el legislador. Por consiguiente, la ley no es combatida en nombre de un derecho perjudicado, ni de otra parte sobre la base del caso concreto al que da lugar su aplicación, pero únicamente con referencia a la "parcialidad" política. La finalidad específica de tal vía procedural no reside por lo tanto en la tutela de los derechos fundamentales ni en la depuración del orden jurídico, pero más bien en la preservación de la autoridad de la ley, dispersando a tal fin las dudas venidas a la luz al momento de la disputa política. Depurar la ley de cada vicio de inconstitucionalidad antes de su entrada en vigor constituye indudablemente el medio más seguro para perseguir tal finalidad. Es igualmente la razón por la cual la forma más pura y por consiguiente el sistema que refleja de la mejor manera el modelo de justicia constitucional basado sobre la ley es encarnado indiscutiblemente por el modelo francés.

El otro grande modelo de justicia constitucional, que tiene por finalidad inmediata la tutela de los derechos y no la depuración del ordenamiento jurídico (que no es que 
una consecuencia derivada y secundaria), encuentra la propia consagración clásica en el sistema del judicial review: los derechos pueden ser invocados en cuanto a su tutela, en vía de acción o excepción, delante de todos los jueces, llamados a protegerlos frente a los demás poderes y también contra la ley, que deja de encontrar aplicación si perjudica a los derechos mismos. A su vez, las decisiones jurisdiccionales pueden ser controladas por el Tribunal Supremo cuya doctrina delimita efectivamente el contenido de los derechos enunciados por la Constitución y que, con base en la doctrina de las political questions, se declara incompetente en solucionar los conflictos cuyo único objeto sea el reparto de las competencias entre los órganos políticos.

Realizaciones de tal modelo son localizadas por el autor -en el ámbito de los sistemas europeos- en la experiencia portuguesa, que combina el control difuso de constitucionalidad con la presencia del Tribunal constitucional, o bien en las experiencias que le permiten al juez de levantar la cuestión de legitimidad constitucional en vía incidental, o bien, por fin, en las experiencias dotadas de adecuados instrumentos de tutela de los derechos fundamentales ante el órgano de justicia constitucional (Alemania y España).

El control concreto de constitucionalidad tiene que considerarse segun RUBIO LLORENTE como instituto "cremallera" situado al límite de los dos modelos pero funcionalmente más cerca del primero.

El objeto de la "controversia" es igualmente la constitucionalidad de la ley y en particular, a pesar del apelativo alemán de control concreto, la constitucionalidad abstracta. Efectivamente, la evaluación que tiene que efectuar la jurisdicción constitucional atañe la validez general de la norma legislativa y no su aplicación a un caso concreto. Sin embargo, la invocación de una cuestión prejudicial que, en todo caso, el juez ordinario levanta ex officio o sobre instancia de las partes, y que, en práctica, significa que el juez considera inconstitucional la norma en objeto, atañe efectivamente un caso concreto y no el enunciado de la ley, pero una interpretación de la misma, es decir una norma de la cual derivan inmediatamente derechos y deberes por las partes del proceso.

Con referencia al dinamismo de los modelos de justicia constitucional explicados y a las líneas de tendencia que los mismos demuestran actualmente de seguir, el autor -en línea con FAVOREU, que percibió ya la tendencia a pasar del control de constitucionalidad de la ley a aquel relativo a su aplicación- cree de poder localizar con suficiente grado de aproximación el trayecto seguido por el proceso de transición de un modelo basado sobre el control de las leyes a un modelo basado sobre la tutela de los derechos: son razón justificativa de ello la fuerza normativa de la Constitución y la fuerza expansiva de los derechos fundamentales; son testimonio de ello la primacía de los instrumentos y las vias jurisdiccionales que aseguran la tutela directa de los derechos fundamentales (el amparo español, el Verfassungsbeschwerde alemán) y la sustancial crisis del recurso de inconstitucionalidad.

Una de las tentativas más elaboradas y acreditadas de superación de las tipologías clásicas de los sistemas de control de constitucionalidad de las leyes es debida a 
F. Fernández Segado [F. Fernández Segado, 2003]. Trazado un paralelo significativo entre la difusión a nivel global de la justicia constitucional, rasgo característico del último cuarto del siglo XX, y la universalidad de la idea de libertad, acompañada por la expansión sin fronteras de un sentimiento común que ve en el respeto de la dignidad del hombre y de los derechos inviolables el principio fundamental y el eje portante de cada sistema de gobierno demócrata y de cada forma de convivencia social civilizada, el autor observa cómo tal expansión haya incidido en medida determinante en la clásica contraposición bipolar a la que han sido reconducidos por largo tiempo el modelo del judicial review of legislation americano y el modelo de la Verfassungsgerichtsbarkeit europeo-kelseniano: el proceso de progresiva e incesante convergencia entre los dos clásicos sistemas de justicia constitucional, la quiebra frontal del modelo kelseniano del "legislador negativo" con el consiguiente papel creativo de los tribunales constitucionales y la enorme heterogeneidad y la generalizada mixtura e hibridación de los actuales sistemas de justicia constitucional, han producido la obsolescencia del clásico bipolarismo "sistema americano-sistema europeo-kelseniano", haciendo necesaria la individuación de una nueva tipología que nos ofrezca una mayor capacidad analítica de los sistemas de justicia constitucional.

Junto a los factores de asimilación entre los dos modelos tradicionales ya señalados por Cappeletti, Fernandez Segado localiza la convivencia, en seno al modelo concentrado, de la cuestión concreta sometida a la decisión de los órganos de justicia constitucional con la abstracción del juicio realizado por los mismos y nota las repercusiones que el caso originario (juicio a quo) produce inevitablemente sobre el juicio constitucional, con particular referencia a la determinación del sentido de los enunciados normativos; alude a la multiplicación y diversificación de la tipología de las pronuncias de las cortes o tribunales constitucionales, que no se limitan más a ejercer un papel meramente negativo, pero que han asumido al revés plenamente la función propia de un legislador positivo a través del recurso a técnicas jurídicas extremadamente diferenciadas típicas del judicial review; nota la disminución de la distancia que anteriormente separaba la eficacia del precedente en América del Norte en virtud del principio del stare decisis y la eficacia de los efectos erga omnes de las sentencias de los tribunales o cortes constitucionales, fenómeno hecho posible por el contenido fundamentalmente interpretativo de las decisiones de estos últimos; subraya la progresiva simetría venida a establecerse, en particular después de la Segunda Guerra Mundial, entre la noción de la Constitución en el sistema norteamericano en cuanto higher law y el sentido de Constitución en cuanto dotada de eficacia normativa superior y que lleva un orden de valores directamente vinculantes los tres poderes del Estado.

Es sobre la base de tales consideraciones que el autor cree definitivamente tramontata la posibilidad de reconducir a la dicotomía clásica de los modelos del control de constitucionalidad experiencias de justicia constitucional connotadas por un grado tan elevado de heterogeneidad, tal de implicar el reconocimiento de nuevas y diferentes variables capaces de proveer una explicación más coherente de las varias fórmulas ofrecidas por los sistemas de control de constitucionalidad. 
Punto de partida y núcleo central de tal estructura analítica es, segun el autor, una dúplice modalidad de control de la constitucionalidad: el control de la ley, realizado en ausencia de una preventiva controversia judicial y de conflictos de intereses subjetivos, y el control de constitucionalidad que tiene lugar durante la aplicación de la ley, caracterizado por requisitos contrapuestos al anterior.

Con referencia a la primera modalidad de control, tienen que acogerse dos nuevas variables: en primer lugar, el momento de efectuación del control, que favorece la diferenciación entre un control a priori, de naturaleza preventiva y un control a posteriori, de naturaleza sucesiva, según el control tenga lugar antes o después lo entrada en vigor de la ley; en segundo lugar, la naturaleza del interés constitucional que se quiere tutelar por el control, teniendo que distinguir entre un control objetivo o en el interés del ordenamiento constitucional general, y un control de competencia que, en el ámbito de un Estado compuesto, resulta finalizado a salvaguardar el sistema constitucional de las competencias atribuido a los diferentes sujetos del Estado-ordenamiento.

Con referencia a la segunda modalidad de control, vienen en relieve tres diferentes variables.

La primera variable atañe la atribución de la competencia a una pluralidad de órganos o a un órgano único, teniendo que diferenciar bajo tal perfil entre control difuso y control concentrado.

La segunda variable atañe la instancia de instauración del control, teniendo que diferenciar cuatro modalidades: el control promovido por un órgano jurisdiccional, como en el caso de la cuestión de legitimidad constitucional; el control promovido por una persona que considere violada la esfera de los propios derechos o intereses legítimos, no necesariamente consubstancial a un procedimiento de recurso directo de inconstitucionalidad; el control promovido por los órganos constitucionales estatales en el marco de un conflicto de atribuciones interorgánico; por fin, el control promovido por los entes autónomos territoriales de un Estado compuesto, finalizado a la reivindicación de la titularidad de competencia injustamente asumida por otro sujeto, en el marco de un conflicto de atribuciones intersubjetivo.

La tercera variable es relativa a la eficacia de las sentencias, teniendo que distinguir según las sentencias desplieguen efectos limitadamente al caso concreto, inter partes, o bien los proyecten con carácter general, erga omnes.

Es sobre la base de tales premisas que el constitucionalista español identifica aquellas que bien podrían considerarse como dos reglas tendenciales dominantes en la jurisdicción constitucional de las alboradas del nuevo siglo: la primera es aquella de la progresiva mutación del control de constitucionalidad de la ley en el control de la aplicación de las leyes; la segunda, la tendencia cada vez más marcada hacia una jurisdicción constitucional basada sobre la tutela de los derechos, lo que no es otro que el resultante obligado del lugar primigenio de la importancia de los derechos y de las libertades en las Constituciones de nuestros tiempos. 
La superación de la alternativa sistema americano-sistema austríaco de control de constitucionalidad de las leyes ha matizado en Italia la posición doctrinal de A. Pizzorusso, caracterizada por un camino teórico-reconstructivo y resultados ampliamente convergentes con respecto de aquéllos evidenciados anteriormente [A. Pizzorusso, 1982, 521 ss.].

Dos son las novedades de mayor relieve que segun el autor han emergido en el ámbito de las experiencias de justicia constitucional en la Europa continental de la segunda posguerra: el papel normativo no solo negativo pero también positivo desarrollado por las pronunciaciones de las cortes o tribunales constitucionales en el marco del control centralizado respecto a disposiciones o normas en vigor; la combinación de la técnica del control incidental de matriz americana con la técnica del control concentrado de matriz austríaca a través del empleo del instituto de la prejudicialidad.

La aproximación entre los recursos propuestos directamente al juez constitucional “concentrado" y los juicios de constitucionalidad propuestos a este último por un juez "difuso" en virtud de la relación de prejudicialidad, permite de otra parte de encontrar una más amplia unidad de los sistemas de garantía de la Constitución si se considera que ambos institutos comportan solamente menores modificaciones de estructura con respecto del "sistema americano", cuya posición se confirma por lo tanto como mucho más central de lo que se piensa generalmente.

El recurso directo (cuando es subordinado, como por lo general, al agotamiento de las vías de recurso ordinario, es decir diferentes de ello) se concreta en efecto en un ulterior grado de jurisdicción que se parece mucho al recurso al Tribunal Supremo americano, que es precisamente no un juez de única, pero de última instancia, mientras que el juicio concentrado-incidental comporta que la decisión de la Corte constitucional funcione prácticamente como una parte componible de la decisión del juez a quo, uniéndose con ella (y con todas las otras relativas a casos análogos en relación a las cuales el problema no sea "agotado") en aquel producto final que es la decisión destinada a devenir cosa juzgada, tal como el punto de derecho de un tribunal supremo se une a la decisión del juez de reenvío.

Es a la luz de la dúplice evolución descrita que el autor considera oportuno superar la contraposición entre sistema americano y sistemas europeos a través de la referencia a aquella entre sistemas concretos y sistemas abstractos de control de constitucionalidad de las leyes. Bajo el perfil técnico, los sistemas concretos vienen ulteriormente articulados a su interior por el autor según la cuestión de legitimidad constitucional sea propuesta al juez junto con una cuestión de aplicación de la ley (como en el caso de los Estados Unidos) o bien sea filtrada por el juez a quo, incluso en consideración de los efectos de las sentencias, determinados en un caso por el principio del stare decisis y, en el otro, del alcance erga omnes de las decisiones. Igualmente bajo el perfil técnico, los sistemas abstractos son distinguidos según el carácter preventivo o sucesivo de la impugnación-decisión y según la tipología de los órganos o los sujetos legitimada a proponer recurso al órgano de justicia constitucional. 
A las mencionadas diferencias de carácter técnico corresponden en la reconstrucción ofrecida por Pizzorusso varias posibilidades de uso de la tutela que las mismas ofrecen.

La tutela asegurada por los sistemas concretos se dirige principalmente a las situaciones jurídicas subjetivas de los ciudadanos y aparece por lo tanto principalmente finalizada a realizar la protección de los derechos fundamentales de libertad que la Constitución garantiza. Por contra la tutela ofrecida por los sistemas abstractos aparece principalmente finalizada a asegurar el respeto de las esferas de competencia de los diferentes entes, en los estados federales o regionales, y de los varios órganos constitucionales, en el ámbito de la organización estatal, mientras para la protección de los derechos individuales o colectivos ella puede ser realizada solamente eventualmente y de reflejo, cuando uno de tales sujetos, por razones de conveniencia política, se haga portador de ella, siempre que las condiciones previstas por el ejercicio de la acción lo permitan.

Consigue de ello que a la contraposición entre sistemas concretos y sistemas abstractos corresponde una latente contraposición entre los sistemas en el ámbito de los cuales el carácter jurídico, y jurisdiccional, del control prevalece sobre el carácter político de ello -que incluso obviamente no puede ser nunca completamente excluido- y los sistemas en el ámbito de los cuales el carácter político es en cambio predominante.

La crítica de $M$. Fromont a la clasificación tradicional de los modelos de control de constitucionalidad mueve de la constatación de la inhabilidad de los mismos a incorporar otras formas de justicia constitucional, en particular las hipótesis de control de las sentencias y los actos administrativos [M. Fromont, 1996]. Tal clasificación tiende a pasar bajo silencio todos los sistemas de justicia constitucional que no corresponden a alguno de los dos modelos, como en numerosas hipótesis de experiencias latinoamericanas o de países europeos como Suiza y Portugal.

Considerando los tres casos de Estados Unidos, Alemania y Francia, el autor advierte cómo el análisis clásico tiende a oponer la experiencia estadounidense a las otras, en cuanto solo estas últimas son dotadas de un sistema de justicia constitucional caracterizado por la presencia de un juez especializado. Considerando el funcionamiento real de la justicia constitucional, en cambio se tendrá que necesariamente yuxtaponer el sistema francés a los otros: Estados Unidos y Alemania otorgan en efecto a un órgano jurisdiccional, respectivamente, Tribunal Supremo y Tribunal constitucional federal, situado en la cumbre del sistema judicial, el control del respeto de la Constitución de parte de todos los tribunales e, indirectamente, de los órganos del Estado. Al contrario el sistema francés atribuye al Consejo constitucional el control de constitucionalidad de proyectos de ley y determinadas cuestiones concernientes al funcionamiento de los poderes públicos.

La diferente clasificación propuesta por Fromont se basa sea sobre la naturaleza de la decisión jurisdiccional que sobre el procedimiento utilizado para llegar a tal decisión, distinguiéndose entre procedimientos concretos y procedimientos abstractos de 
control de constitucionalidad. Los primeros se basan en una lógica subjetiva que asume los derechos de la persona cuál eje central de referencia, mientras que los segundos corresponden a una lógica objetiva que atribuye prioridad al interés del Estado. No se desconoce sin embargo, de parte del constitucionalista francés, la existencia de casos en los cuales se encuentra la combinación de procedimientos objetivos y concretos, además de procedimientos subjetivos y abstractos: se origina una tipología articulada en cinco grupos de experiencias. Algunos ordenamientos únicamente acogen procedimientos subjetivos y concretos (es el caso de Estados Unidos); otros contemplan procedimientos subjetivos y concretos, admitiendo sin embargo también procedimientos abstractos y mixtos (es el caso de Alemania); algunos ordenamientos son caracterizados principalmente por la presencia de procedimientos mixtos (lo que ocurre en Italia); otros predominantemente recurren a procedimientos objetivos y abstractos, como en el caso de Austria; otros, por fin, presentan exclusivamente procedimientos objetivos y abstractos (el arquetipo es ofrecido por la experiencia francesa).

La expansión global de la justicia constitucional, en particular después de la Segunda Guerra Mundial, ha sido acompañada de la proliferación de los modelos y de su recíproca hibridación, tanto de inducir una pluralidad de estudiosos a inventar nuevos esquemas clasificatorios y nuevas categorías, cuya ductilidad a los fines de la acogida a su interior de experiencias de reciente emersión no se ha revelado suficiente. La multiplicación por la doctrina de los modelos de justicia constitucional, en otras palabras, no facilita el estudio de ellos. Para reducir los márgenes de aproximación para la comprensión de los trayectos evolutivos seguida por el modelo americano y el modelo europeo parece imprescindible colocarse en una óptica que tienda a recobrar la noción de protección de la Constitución y tal de evidenciar la múltiple función desarrollada por los órganos de justicia constitucional a tal fin, además de privilegiar una perspectiva no eurocéntrica, que identifique cual metodología fundamental la originalidad y la matriz norteamericana de la justicia constitucional y en particular del control de constitucionalidad de las leyes [H. J. ABRAHAM, 1993, 271 ss.].

Sólo si se reconoce la antecedencia del modelo estadounidense, cuanto a su elaboración, a su desarrollo y a su aplicación, bajo el aspecto doctrinal, normativo y jurisprudencial, con respecto del modelo europeo, puede entenderse la naturaleza derivada del segundo con respecto de lo primero y el fenómeno de tendencial asimilación de los dos modelos.

No tiene que olvidarse, en efecto, que el sistema de justicia constitucional nace y se desarrolla en los Estados Unidos cual complejo funcional caracterizado por el ejercicio de parte del Tribunal Supremo, junto al judicial review, de competencias típicamente pertinentes a la jurisdicción federal y a la jurisdicción de las libertades, luego objeto de consideraciòn al momento de la creación de los sistemas europeos. Es más bien sobre la base de tal caracterización plural que se han evidenciado correctamente 
tres períodos de influencia del sistema americano de justicia constitucional respecto a experiencias extranjeras, entre las cuales aquellas europeas [D. P. Kommers, 1976, 55 ss.]: es pues el modelo americano que irradia en el curso de las décadas recientes aquel europeo, y no viceversa. Del hecho que el modelo europeo se configura cuál mera versión aplicativa del modelo americano se tiene sin embargo confirmación si se observa -con referencia al control de constitucionalidad de las normas- la progresiva recuperación de parte de lo primero de las connotaciones originales del prototipo de origen, casi a absorber la anomalía y la desviación de lo mismo concebida al momento de la configuración de órganos y procedimientos predispuestos para el control centralizado de constitucionalidad.

Soy testimonio de ello varios factores, que sirven de inequívocos índices reveladores del fenómeno: en los "sistemas mixtos", el arquetipo es aquel estadounidense, los elementos meramente hibridantes aquellos europeos; si se considera la experiencia italiana -pero es observación que puede repetirse con referencia a una amplia pluralidad de ordenamientos- el Tribunal de casación desarrolla inequívocas funciones de justicia constitucional coparticipando, junto a la Corte constitucional, a la definición o bien redefinición del orden constitucional en su complejo, aptitud que se manifiesta con particular evidencia a la hora de la elaboración de nuevos derechos, pero que se traduce también, por ejemplo, en la delineación de los contornos funcionales de órganos constitucionales, en la individuación de la posición de las diversas fuentes en el sistema de las fuentes del derecho, en la consideración de la relación, en el sentido de la inmediata aplicabilidad, de las fuentes internacionales con el ordenamiento nacional, eso eventualmente en competencia o bien en disidencia con respecto de la doctrina del órgano de justicia constitucional: en tales casos emerge un sistema bicéfalo de justicia constitucional, basado en paralelismos y asimetrías que denotan, según las diversas hipótesis, convergencias y disensos entre Tribunal de casación y Corte constitucional y tal de no legitimar una apriorística y simplista opción a favor de la segunda con respecto del primero cuanto al predominio del peso específico cualitativo y cuantitativo revestido por los dos órganos para la construcción del bloque de constitucionalidad considerado en su conjunto; el juez ordinario, en cuanto introductor necesario del juicio de legitimidad constitucional en via incidental, siempre desarrolla un primer segmento del control de constitucionalidad cuando averigua la subsistencia de los requisitos de la relevancia y de no manifiesta falta de fundamento de la cuestión de legitimidad constitucional; el proceso constitucional incidental presenta frecuentemente, junto a un carácter de objetivo interés por el entero ordenamiento, un carácter subjetivo en cuanto finalizado a la tutela de situaciones jurídicas subjetivas de las partes del proceso a quo; en seno al juicio en vía incidental, el hecho o el objeto del juicio a quo inevitablemente irradian la misma influencia respecto al órgano de justicia constitucional; los órganos de justicia constitucional europeos han desarrollado una amplia tipología de decisiones que asimilan su papel al del Tribunal Supremo estadounidense (en Italia, las sentencias interpretativas de rechazo y las de inadmisibilidad; el juez ordinario puede desaplicar, y el juez administrativo puede anular, normas jurídicas inconstitucionales contenidas en fuentes de rango sublegislativo, por ejemplo reglamentos del poder ejecutivo); en los ordenamientos de los Países miembros de la Unión Europea, los jue- 
ces de cada grado deben desaplicar normas nacionales contrastantes con el derecho comunitario, y por lo tanto con las normas constitucionales que afirman o justifican su primacía, y aplicar las normas comunitarias; igualmente en ámbito comunitario, los jueces de los Estados miembros pueden (si de última instancia, deben) suspender el proceso y recurrir a la Corte de Justicia de la Unión Europea con un recurso en vía prejudicial si tienen dudas acerca de la adecuada transposición del derecho comunitario en el derecho interior por el legislador nacional.

En conclusión, se puede hablar de convergencia del modelo norteamericano y el modelo europeo de justicia constitucional sólo si se tiene claro que el segundo, variable de lo primero, tiende a evolucionar refluyendo en amplia medida dentro de lo primero. En tal sentido, cuando se aborda al estudio de los sistemas de justicia constitucional, parece más correcto adoptar un contexto de referencia unitaria, predominantemente caracterizado al propio interior del ejercicio de funciones de protección de la Constitución de naturaleza y finalización, si bien no exclusivamente, objetiva, de interés del entero ordenamiento (ej., la resolución de los conflictos interorgánicos y intersubjetivos), y de funciones de protección de la Constitución de naturaleza y finalización predominantemente, si bien no exclusivamente, subjetiva (ej., los recursos directos de constitucionalidad a tutela de los derechos fundamentales o bien a la protección de los mismos que se realiza durante el juicio en vía incidental).

III

El papel y las funciones de los órganos de justicia constitucional en las democracias contemporáneas, y correlativamente las razones justificativas de su legitimación en seno a las mismas, pueden devenir objeto de valoración en una perspectiva parcial y sectorial, coincidente con el control de constitucionalidad de las leyes, o bien en una perspectiva sistémica, tal de abrazar las funciones de los órganos mismos en su complejo.

Si se adopta como parámetro de referencia la función consistente en el control de la legitimidad constitucional de las leyes, se nota cómo el papel desarrollado por los órganos de justicia constitucional en tal sentido se realiza en la tendencial avocación a una sede diferente y ulterior con respecto de aquella política del proceso de reconocimiento y desarrollo de los valores constitucionales, en la contribución provista por el juez constitucional a la aclaración del contenido de las normas constitucionales a través de una obra hermenéutica que, según las diferentes hipótesis, acompaña, integra, enmienda o incluso reemplaza las opciones efectuadas por el legislador.

Se realiza, en el modo descrito, una ponderación complementaria y subsidiaria de los intereses emergentes en seno a la sociedad con respecto de cada ámbito material que, en las hipótesis más calificadas, se traducen en un tipo de drenaje axiológico de parte del juez constitucional, que contribuye a la individuación de los principios fundamentales y de los valores basicos a los cuales tiene que necesariamente conformarse el legislador que quiera regular o realizar la adecuación normativa de una determinada materia. 
Los órganos de justicia constitucional, por otro lado, no faltan de proponerse, bajo el perfil indicado, cuales guardianes del respeto de las reglas constitucionales sobre las cuales se ha realizado el consenso: la función desarrollada por el juez constitucional en la óptica descrita, por tanto, no es finalizada únicamente a la realización del consenso, sino también a la preservación, a la salvaguardia y la consolidación del consenso sobre las reglas mismas. Favoreciendo, completando o censurando las opciones normativas adoptadas por el legislador, los órganos de justicia constitucional además ejercen, en el sentido precisado, una función de legitimación del contenido de los actos legislativos, cuya verificación de parte del juez constitucional se traduce en un tipo de certificación de la constitucionalidad en las hipótesis de control de legitimidad constitucional en vía preventiva, como en la experiencia francesa, o en la determinación y atestación de la conformidad a la Constitución de la proposición normativa forjada por el legislador, por lo tanto en la legitimación del contenido que caracteriza la proposición misma. Tal determinación, además, se pone particularmente incisiva donde a las sentencias constitucionales sea reconocida fuerza de ley.

Los órganos de justicia constitucional cuentan por lo general con una articulada tipología de decisiones que permite al juez constitucional modular y variar el impacto político de las mismas sentencias, que se vuelven instrumento utilizado con diferente ductilidad según el grado de injerencia dentro de la esfera discrecional del legislador que el juez mismo quiere alcanzar.

La morfología de las sentencias constitucionales resulta en tal modo extremadamente variada y es caracterizada por la presencia, en seno al arco -cuyos polos extremos son representados por las sentencias de mera estimación o rechazo de la cuestión de constitucionalidad- de tipos de decisiones que hacen extremadamente elásticos los márgenes de operatividad de los titulares del poder legislativo en su obra de regulación o reforma de las diversas materias. La disponibilidad del amplio instrumentario jurisprudencial mencionado, por otro lado, induce el juez constitucional a concebir en llave igualmente dúctil y elástica el principio del self-restraint y a relativizar el principio, al primero estrechamente conexo, de la abstención de decisiones que atañen o únicamente rocen political questions de incumbencia del legislador.

La tendencia seguida por los órganos de justicia constitucional se realiza más bien en la configuración de parte de los mismos de hipótesis y soluciones alternativas a favor del legislador que quiera poner mano a la regulación o a la reforma de diferentes caos, aunque dentro de un cuadro determinado por el juez constitucional y a través de la aposición de límites generalmente precisos, por lo general acompañada por la advertencia de proveer en el sentido auspiciado o de la amenaza de sancionar durante los procesos constitucionales opciones diferentes eventualmente preferidas por el legislador en sede parlamentaria. Del empleo del instrumentario jurisprudencial en el sentido descrito trasluce el intento del juez constitucional de valorizar el papel y las funciones de moderación, de crítica y de decantación del proceso decisional desarrollado por las minorías, los grupos de interés, los sujetos exponenciales de intereses colectivos o difusos, que resultaría de otro modo carente de interlocutores fundamen- 
tales, que encuentran en la justicia constitucional una sede de representación de las mismas instancias.

Es sobre la base de tales observaciones que emerge la necesidad de contemplar las jurisdicciones constitucionales no sólo como "guardianes negativos" de la Constitución, llamados a depurar el orden jurídico de las normas inconstitucionales creadas por los demás poderes del Estado [L. Garlicki-W. Zakrzewski, 1985, 31] -lo que tendería a subrayar de ello la sola dimensión estática- pero también como sus guardianes activos, que en su misma dimensión dinámica son llamados a desarrollar una función creadora de principios, valores y derechos constitucionales, una función de arbitraje en el ámbito de los conflictos que intervengan entre sujetos u órganos constitucionales, en general una función de rectificación del sistema considerado en su conjunto finalizada a la búsqueda de dos objetivos fundamentales: Ilenar el hiato que existe a veces entre derecho y sociedad y proteger la democracia [A. Barak, 2006, 231].

Tal función de reconducción del derecho a la justicia se materializa en la censura de la obra del legislador, es acompañada por la identificación de principios, valores y derechos constitucionales que pone al día y detalla las opciones originariamente efectuadas por el constituyente, procediendo donde sea necesario a la ponderación y al consiguiente balance de los intereses, bajo el aspecto de la parte dogmática, además sobre aquel organizativo y ordinamental de las Constituciones, y eventualmente es integrada por el ejercicio de ulteriores funciones finalizadas en su conjunto a garantizar la perpetuación de la Constitución en el tiempo (resolución de los conflictos interorgánicos e intersubjetivos, control sobre los partidos políticos, etcétera).

La fuente de legitimación de funciones de tal entidad cualitativa y cuantitativa tiene que necesariamente ser buscada al más alto nivel y tiene que identificarse con el poder constituyente y con la Constitución que el mismo ha implantado. La justicia constitucional goza de legitimación democrática en cuanto se encuentra contemplada en el seno de una Constitución democrática surgida según un procedimiento democrático. Los constituyentes que, sobre todo después de 1945, han acogido en el seno de las Constituciones contemporáneas uno de los modelos de justicia constitucional y en particular el control de legitimidad constitucional de las leyes, han concebido la perspectiva de que los órganos de justicia constitucional como sus comisarios para el futuro, otorgándoles el mandato, siempre revocable, teniendo lugar un acontecimiento calificable según la terminología de ACKERMAN como momento constitucional, a proteger, adecuar y poner al día las opciones seguidas a la hora de la adopción del acto fondativo del ordenamiento. Los órganos de justicia constitucional participan más cerca de los demás poderes constituidos de la naturaleza típica del poder constituyente.

Los constituyentes toman acto, a partir de la segunda posguerra, de la insuficiencia del circuito representativo tradicional a asegurar idónea representación a la pluralidad de los intereses emergentes en el seno de la sociedad y de la inadecuación de la ley, que se convirtió - como ya Bachof y Forsthoff advirtieron- cada vez más en contingente y expuesta a las humorales aspiraciones de minorías ocasionales, a seleccionarlos y 
a asegurar apropiada tutela no retrocediendo con respecto de los tradicionales canones de la generalidad y abstracción de las normas. Parlamentos divididos, perezosos, contemporizadores e incapaces de proveer idónea declinación técnica a los intereses representados, además del progresivo comportarse de la ley cual ley-medida, son factores capaces de erosionar desde sus fundamentos el Estado constitucional demócrata y de derecho.

La justicia constitucional también nace en consecuencia de la profunda crisis de identidad y funcional que asedia la ley y los parlamentos en las democracias contemporáneas y de la voluntad de los constituyentes de preservar la democracia y la justicia más allá de las transitorias contingencias que tienden a adquirir las formas de un derecho precario.

\section{BIBLIOGRAFÍA ESENCIAL}

AA.VV., (2004). Estado de derecho y la justicia constitucional en el siglo XXI, (Cochabamba, Kipus).

AA.VV., (2006). Reforma de la Constitución y control de constitucionalidad, (Bogotá, Pontificia Universidad Javeriana).

Авraham, H.J., (1993). The Judicial Process. An Introductory Analysis of the Courts in the United States, England and France, (Oxford, Oxford University Press).

Acosta SÁnchez, J.A., (1998). Formación de la Constitución y jurisdicción constitucional, Madrid, Tecnos.

Adams, J., (2006). Una difesa delle Costituzioni di governo degli Stati Uniti d'America, in IDEM, Rivoluzioni e costituzioni, trad. it., (Napoli, Guida).

Ahumada Ruiz, M., 2005. La jurisdicción constitucional en Europa, (Madrid, Thomson). Alexy, R., (1986). Theorie der Grundrechte, Suhrkamp, Frankfurt a.M.

BaCHOF, O., (1959), Grundgesetz und Richtermacht, Mohr, Tübingen, disponible en lengua española, (1963). Jueces y Constitución, (Madrid, Taurus).

Balaguer Callejón, M.L., (1997). Interpretación de la Constitución y ordenamiento jurídico, (Madrid, Tecnos).

Barak, A., (2005). Constitutional interpretation, in F. Mélin-Soucramanien (a cura di), L'interpretétation constitutionnelle, (Paris, Dalloz), 91 ss.

BARAK, A., (2006). L'exercice de la fonction jurisdictionnelle vu par un juge: le rôle de la Cour suprême dans une démocratie, in Revue française de Droit constitutionnel, 227 ss.

Barber S.A.-Fleming, J.E., (2007). Constitutional Interpretation, (New York, Oxford University Press).

Battaglini, M., (1957).Contributi alla storia del controllo di costituzionalità delle leggi, (Milano, Giuffrè).

BatTAGlini, M., (1962). Contributo allo studio comparato del controllo di costituzionalità: i paesi che non hanno controllo, in Riv. trim. dir. pubbl., 1962, 663 ss.

BAZAN, V. (a cura di), (1996). Desafíos del control de constitucionalidad, (Buenos Aires, Ciudad Argentina).

BeatTy, D. M., (2004). The Ultimate Rule of Law, (New York, Oxford University Press). 
Bianchi, A.B., (2002). Control de constitucionalidad, 2 voll., (Buenos Aires, Ábaco).

BIN, R., (1992). Diritti e argomenti. Il bilanciamento degli interessi nella giurisprudenza costituzionale, (Milano, Giuffrè).

Blanco Valdés, R., (1997). Il valore della Costituzione, (Padova, Cedam).

Blume FortinI, E., (1996). El control de la constitucionalidad, (Lima, Ersa).

BÖCKENFÖRDE, E. W., (2006). Giurisdizione costituzionale. Questioni strutturali, organizzazione, legittimazione, in IDEM, Stato, costituzione, democrazia, (Milano, Giuffrè).

BonetrI, P., (2006). Terrorismo, emergenza e costituzioni democratiche, (Bologna, il Mulino).

Brage Camazano, J., (2005). La jurisdicción constitucional de la libertad, (México, Porrúa).

Brewer-Carias, A.R., (1996). Instituciones políticas y constitucionales. Tomo VI-Justicia constitucional, (Caracas, Editorial Jurídica Venezolana).

Breyer, S., (2005). Active Liberty. Interpreting Our Democratic Constitution, (New York, Vintage Books).

ButtÀ, G., (1988). John Adams e gl'inizi del costituzionalismo americano, (Milano, Giuffrè).

Calamandrei P., (1950). La illegittimità costituzionale delle leggi nel processo civile, (Padova, Cedam).

Cappelletti, M., (1968). Il controllo giudiziario di costituzionalità delle leggi nel diritto comparato, Milano, Giuffrè.

Cappelletti, M., (1955). La giurisdizione costituzionale delle libertà, (Milano, Giuffrè).

Cappelletti, M.-Adams, J.C., (1966). Judicial Review of Legislation: European Antecedents and Adaptations, in Harvard Law Review.

Castañeda Otsu, S., (2004). Derecho procesal constitucional, Cochabamba, Kipus.

Chagnollaud, D. (a cura di), (2003). Aux origines du contrôle de constitutionnalité. $X$ VIII- $X X$ siècle, (Paris, LGDJ).

CHeLI, E., (1996). II giudice delle leggi, (Bologna, il Mulino).

CHESSA, O., (1998). Bilanciamento ben temperato o sindacato esterno di ragionevolezza? Note sui diritti inviolabili come parametro del giudizio di costituzionalità, in Giur. cost.

Corrado, M.L., (2005). Comparative Constitutional Review, (Durham, Carolina Academic Press).

Corwin, E. S., (1929). L'idea di "legge superiore” e il diritto costituzionale americano, trad. it., (Venezia, Neri Pozza).

CrISAfulli, V., (1978). La Corte costituzionale ha vent'anni, in N. Occhiocupo (a cura di), La Corte costituzionale tra norma giuridica e realtà sociale, (Bologna, il Mulino).

CRUz Villalón, P., (1987). La formación del sistema europeo de control de constitucionalidad, (Madrid, CEC).

De Moraes, A., (2000). Jurisdição constitucional e tribunais constitucionais, (São Paulo, Atlas).

De Morais, C.B., (2002). Justiça constitucional, (Coimbra,Coimbra Editora).

De Vergottini, G. (a cura di), (2000). Giustizia costituzionale e sviluppo democratico nei Paesi dell'Europa centro-orientale, (Torino, Giappichelli).

Dermizaky Peredo, P., (2003). Justicia constitucional y estado de derecho, (Cochabamba, Alexander).

Di Gregorio, A., (2004). L La giustizia costituzionale in Russia. Origini, modelli, giurisprudenza, (Milano,Giuffrè). 
Diallo, I., (2004). Á la recherche d'un modale africain de justice constitutionnelle, in Annuaire International de Justice Constitutionnelle, 93 ss.

Díaz Revorio, F. J., (2004). La Constitución abierta y su interpretación, Lima, Palestra.

Dietze, G., (1958). America and Europe. Decline and Emergence of Judicial Review, in Virginia Law Review, 44, (1958, (1233 ss.

Dworkin, R., (1982). I diritti presi sul serio, trad. it, (Bologna, il Mulino).

Eberl, M., (2006). Verfassung und Richterspruch, (Berlin, De Gruyter).

EINAudi, M., (1931). Le origini dottrinali e storiche del controllo giudiziario sulla costituzionalità delle leggi negli Stati Uniti d'America, (Torino, Memorie dell'Istituto giuridico).

Ely, J. H., (1981). Democracy and Distrust. A Theory of Judicial Review, (Cambridge, Harvard University Press).

Espin Templado, E.-F.J. Díaz Revorio (a cura di), (2000). La justicia constitucional en el estado democratico, (Valencia, Tirant lo Blanch).

Espinosa-Saldaña Barrera, E. (a cura di), (2005). Derechos fundamentales y derecho procesal constitucional, (Lima, Jurista Editores).

Espinosa-SaldAÑa Barrera, E., (2003). Jurisdicción constitucional, impartición de justicia $y$ debido proceso, (Lima, ARA Editores).

Favoreu, L., (1986). Europe occidentale, in L. Favoreu - J.-A. Jolowicz (a cura di), Le contrôle juridictionnel des lois, (Paris, Economica), 45 ss.

Favoreu, L., (1996). Les Cours constitutionnelles, (Paris, PUF).

FAvoreu, L., (2003). Los contenciosos constitucionales: aproximación teórico comparada, in E. Ferrer Mac-Gregor (a cura di), Derecho procesal constitucional, vol. I, (Mexico, Porrúa), 139 ss.

Favoreu, L., (1997). Los tribunales constitucionales, in D. Garcia Belaunde - F. Fernandez SEGADO (a cura di), La jurisdicción constitucional en Iberoamérica, (Madrid, Dykinson), 95 ss.

FEDE, F., (2001). La giurisdizione costituzionale nelle repubbliche europee della ex Unione sovietica, (Padova, Cedam).

FernÁndez SeGAdo, F., (2003). La jurisdicción constitucional ante el siglo XXI. La quiebra de la bipolaridad "sistema americano-sistema europeo-kelseniano" y la búsqueda de nuevas variables explicativas de los sistemas de control de constitucionalidad, Córdoba.

FernÁndez Segado, F., (2003). La jurisdicción constitucional en América latina. Evolución y problemática desde la independencia hasta 1979, in E. Ferrer MAc-Gregor (a cura di), 2003, Derecho procesal constitucional, vol. I, (México, Porrúa), 149 ss.

FERnÁNDEZ SEGAdo, F., (2009). La justicia constitucional. Una visión de derecho comparado, 3 voll., (Madrid, Dykinson).

Ferrari, G. F. (a cura di), (2006). Corti nazionali e Corti europee, (Napoli, ESI).

Ferrari, G.F.-Gambaro A. (a cura di), (2006). Corti nazionali e comparazione giuridica, (Napoli, ESI).

Ferreira Mendes, G., (1999). Jurisdição constitucional, (São Paulo, Saraiva).

Ferrer Mac-Gregor, E. (a cura di), (2003Derecho procesal constitucional, 4 voll., (MéxiCo, Porrúa).

Ferrer-Mac Gregor, E., (2002). Los tribunales constitucionales en Iberoamérica, (México, Fundap). 
Ferreira Mendes, G., (2002). Direitos fundamentais e controle de constitucionalidade, (São Paulo, Celso Bastos).

Fix-Zamudio, H. (1999). Breves reflexiones sobre el concepto y el contenido del derecho procesal constitucional, in Anuario Iberoamericano de Justicia Constitucional, 89 ss., ora anche in E. Ferrer Mac-Gregor (a cura di), 2003, Derecho procesal constitucional, I, (Mexico, Porrúa), 269 ss.

Fix-Zamudio, H.-Ferrer Mac-Gregor, E. (a cura di), (2006). El derecho de amparo en el mundo, (México Porrúa).

Fix-Zamudio, H.-Valencia Carmona, S., (2008). Derecho constitucional mexicano y comparado, (México,Porrúa).

Fromont, M., (1996). La justice constitutionnelle dans le monde, (Paris, Dalloz).

García Belaunde, D., (2000). De la jurisdicción constitucional al derecho procesal constitucional, (Lima, Grijley).

García Belaunde, D., (2001). Derecho procesal constitucional, (Bogotá, Temis).

Garvey, J. H., Aleinikoff, T. A., Farber, D. A., (2004). Modern Constitutional Theory: A Reader, Thomson.

Ginsburg, T., (2003). Judicial Review in New democracies. Constitutional Courts in Asian Cases, (Cambridge, Cambridge University Press).

Gomes Canotilno, J. J., (2009). Direito Constitucional, (Coimbra, Almedina).

Gomes Canotilno, J.J., (2003). Direito constitucional e Teoria da Constituição, (Coimbra, Almedina).

GonZÁlez Rivas, J.J., (2001). Análisis de los sistemas de jurisdicción constitucional, (Madrid, CEC).

González-Trevijano SÁnchez, P.J., (2000). El Tribunal constitucional, (Elcano, Aranzadi).

GozAINI, O. A., (1994). La justicia constitucional, (Buenos Aires, Depalma).

GrANt, J. A. C., (1963). El control jurisidiccional de la constitucionalidad de las leyes. Una contribución de las Américas a la ciencia política, (México, Unam).

Grewe, C.-Jouanjan, O.-Maulin, E.-Wachsmann, P. (a cura di), (2005). La notion de "justice constitutionnelle", (Paris, Dalloz).

Häвerle, P., (2005). Europäische Verfassungslehre, III ed., (Baden-Baden, Nomos Verlag).

HÄBERLE, P., (2005). Lo Stato costituzionale, Istituto della Enciclopedia Italiana Treccani, Roma,

HÄBERLE, P., (2006). Verfassungsgerichtsbarkeit in der offenen Gesellschaft, in VAN OOYEN, R. C.-Möllers, M. H. W. (a cura di), Das Bundedsverfassungsgericht im politischen System, Verlag für Sozialwissenschaften, Wiesbaden, 35 ss.

HÄBERle, P., (1980). Verfassungsgerichtsbarkeit zwischen Politik und Rechtswissenschaft, Königstein.

Haltern, U., (1998). Verfassungsgerichtsbarkeit, Demokratie und Misstrauen, (Berlin,Duncker \& Humblot).

Hamilton, A.- Madison, J.- Jay, J., (1997). Il Federalista, trad. it., (Bologna, il Mulino).

Harutyunyan G.-Mavèlè A., (1999). The Constitutional Review and Its Development in The Modern World. A Comparative Constitutional Analysis, (Erevan-Lubiana).

Horn, H.R.-Weber, A. (a cura di), (1989). Richterliche Verfassungskontrolle in Lateinamerika, Spanien und Portugal, (Baden-Baden, Nomos).

KelSEN, H., (1981. La garanzia giurisdizionale della Costituzione (La giustizia costituzionale), in Kelsen, H., La giustizia costituzionale, trad. it., (Milano, Giuffrè), 143 ss. 
Kommers, D. P., (1976). Judicial review. Its influence abroad, in ANNALS of the American Academy of Political and Social Science, Vol. 428, No. 1, 52 ss.

Koopmans, T., (2003). Courts and Political Institutions, Cambridge, (Cambridge University Press).

KorINEK, K., (2000). Grundrechte und Verfassungsgerichtsbarkeit, (Wien-New York, Springer).

LAMBERT, E., (1996). Il governo dei giudici e la lotta contro la legislazione sociale negli Stati Uniti, trad. it., (Milano, Giuffrè).

LANDA, C., (2003). Teoría del derecho procesal constitucional, (Lima, Palestra).

LösinG, N., (2002). La jurisdicciòn constitucional en Latinoamérica, (Madrid, Dykinson).

Luther, J.-Romboli, R.-TARCHI, R. (a cura di), (2000). Esperienze di giustizia costituzionale, 2 voll., (Giappichelli, Torino).

Marcic, R., (1963). Verfasssung und Verfassungsgericht, (Springer Verlag, Wien).

MASSIAS, J.P. (a cura di), (1998). Justice constitutionnelle et transition democratique en Europe de l'Est, Presses Universitaires de la Faculté de Droit de Clermont Ferrand (Clermont L.G.D.J. Ferrand).

Massing, O., (2005). Politik als Recht-Recht als Politik, (Baden-Baden, Nomos Verlag). Mazza, M., (1999). La giustizia costituzionale in Europa orientale, (Padova, Cedam).

McWhinney, E., (1962). Constitutionalism in Germany and the Federal Constitutional Court, (Leiden, Sythoff).

McWhinney, E., (1956). Judicial Review in the English-Speaking World, (Toronto, University of Toronto Press).

McWhinney, E., (1986). Supreme Courts and Judicial Law-Making: Constitutional Tribunals and Constitutional Review, (Dordrecht, Martinus Nijhoff Publishers).

Mezzetti, L. - Belletti, M. - D’Orlando, E. - Ferioli, E., (2007). La giustizia costituzionale, (Padova, Cedam).

Mezzettı, L. (a cura di), (2009). Sistemi e modelli di giustizia costituzionale, (Padova, Cedam).

Mezzettı, L., (2006). Modelli di giustizia costituzionale e controllo di costituzionalità in America Latina, in Ferrari G.F.-Gambaro A. (a cura di), (2006). Corti nazionali e comparazione giuridica, (ESI, Napoli), 533 ss.

Morrone, A., (2001). Il custode della ragionevolezza, (Milano).

Neal Tate, C.-Vallinder, T. (a cura di), (1995). The Global Expansion of Judicial Power, (New York New York University Press).

Noguelra, H., (2004). La jurisdicción constitucional y los tribunales constitucionales de Sudamérica en la alborada del siglo XXI, (México, Porrúa).

Olivetti, M.-GroppI, T. (a cura di), (2003). La giustizia costituzionale in Europa, (Milano, Giuffrè).

Ortecho Villena, V.J., (2002). Jurisdicción y procesos constitucionales, (Lima, Rodhas).

Ould Boubout, , A. S., (1997). Les juridictions constitutionnelles en Afrique, in Annuaire International de Justice Constitutionnelle, 31 ss.

Palomino Manchego, J. F., (2003). Los orígenes de los tribunales constitucionales en Iberoamérica, in E. Ferrer MAc-GreGor (a cura di), Derecho procesal constitucional, vol. I, (México, Porrúa), 407 ss.

Palomino Manchego, J. F.-Remotti Carbonell, J.C. (a cura di), (2002). Derechos Humanos y Constitución en Iberamérica, (Lima, Grijley). 
Pasquino, P., (2002). Tipologia della giustizia costituzionale in Europa, in Riv. trim. dir. pubbl., 359 ss.

Pasquino, P.-RAndAzzo, B. (a cura di), (2006). La giustizia costituzionale e i suoi utenti, (Milano, Giuffrè).

Pegoraro, L., (2007). Lineamenti di giustizia costituzionale comparata, (Torino, Giappichelli).

Perez Royo, J.-Urìas Martinez, J.P.-Carrasco Durán, M. (a cura di), (2006). Derecho constitucional para el siglo XXI, 2 voll., (Elcano, Thomson-Aranzadi).

Perry, M. J., (1994). The Constitution in the Courts, (New York, Oxford University Press). Perry, M. J., (1982). The Constitution, the Courts and Human Rights. An Inquiry into the Legitimacy of Constitutional Policymaking by the Judiciary, (New Haven-London, Yale University Press).

Piperno, C., (1991). La Corte costituzionale e il limite di political question, (Milano,Giuffrè). Pızzorusso, A., (1982). I sistemi di giustizia costituzionale: dai modelli alla prassi, in Quaderni costituzionali, $521 \mathrm{ss.}$

ProchÁzka, R., (2002). Mission Accomplished: on Founding Constitutional Adjudication in Central Europe, (Budapest, New York, CEU Press).

Ramos Tavares, A., (2005). Teoria da justiça constitucional, (São Paulo, Saraiva).

Rivera Santivañez, J.A., (2004). Jurisdicción constitucional, (Cochabamba, Kipus).

Rомвоц R. (a cura di), (1994). La tutela dei diritti fondamentali davanti alle Corti costituzionali, (Giappichelli, Torino).

Rousseau, D. (a cura di), (1998). La démocratie continue, (Bruxelles, LGDJ-Bruylant). Rousseau, D., (2006). Droit du contentieux constitutionnel, (Paris, Montchrestien).

Rubio Correa, M., (2005). La interpretación de la Constitución según el Tribunal Constitucional, (Lima, Pontificia Universidad Católica del Perú).

Rubio Llorente, F., (1992). "Seis tesis sobre la jurisdicción constitucional en Europa”, en Revista Española de Derecho Constitucional, 35, pp. 9 y ss.

Rubio Llorente, F., (1996). "Tendances actuelles de la juridiction constitutionnelle en Europe”, in Annuaire International de Justice Constitutionnelle, 9 ss.

Ruggeri, A.-Spadaro, A., (2009). Lineamenti di giustizia costituzionale, (Giappichelli, Torino).

SAdurSki, W., (2002). Constitutional Justice, East and West: Introduction, in IDEM, Constitutional Justice, East and West. Democratic Legitimacy and Constitutional Courts in Post-Communist Europe in a Comparative Perspective, 8Kluwer, Den Haag), 1 ss.

SAGüés, N., (2002). Derecho procesal constitucional, (Buenos Aires, Ed. Astrea).

SAGüés, N., (1984). Derecho procesal constitucional. Recurso extraordinario, (Buenos Aires, Depalma).

Schlothauer, R., (1979). Zur Krise der Verfassungsgerichtsbarkeit, (Suhrkamp, Frankfurt a.M.).

Sснмiтt, C., (1981). Il custode della Costituzione, trad. it., (Milano, Giuffrè).

Schwartz, H., (2000). The Struggle for Constitutional Justice in Post-Communist Europe, University of Chicago Press, Chicago

SolA, J. V., (2001). Control judicial de constitucionalidad, (Buenos Aires, AbeledoPerrot).

StARCK, C. (a cura di), (2004). Fortschritte der Verfassungsgerichtsbarkeit in der Welt, III, (Baden-Baden,Nomos) (I 2004, II 2006). 
Treanor, W. M., (2005). Judicial Review before Marbury, in Stanford Law Review, 58, 455 ss.

TriBe, L.H., Dorf, M. C., (2005). Leggere la Costituzione. Una lezione americana, trad. it., (Bologna, il Mulino).

VAN OOYEn, R.C.-Möllers, M.H.W. (a cura di), (2006). Das Bundesverfassungsgericht im politischen System, (Wiesbaden, Verlag für Sozialwissenschaften).

Vanossi, J.R., (2000). Teoría constitucional, 2 voll., (Buenos Aires, Depalma).

Verdussen, M. (a cura di), (1997). La Justice Constitutionnelle en Europe Centrale, (Paris, Bruxelles, L.G.D.J.).

Von BRÜNNECK, A., (1988). Le contrôle de constitutionnalité et le legislateur dans les democraties occidentales, in Annuaire International de Justice Constitutionnelle, p. 15 ss.

VorläNDER, H., (2006). Deutungsmacht - Die Macht der Verfassungsgerichtsbarkeit, in IDEM (a cura di), Die Deutungsmacht der Verfassungsgerichtsbarkeit, (Wiesbaden, Verlag für Sozialwissenschaften), 9 ss.

Weber, A., (2003). "Notes sur la justice constitutionnelle comparée: convergences et divergences", in Annuaire International de Justice Constitutionnelle, 29 ss.

ZaGrebelsky, G., (2009). La legge e la sua giustizia, (Bologna, il Mulino). 